\title{
Properties of subvisible cirrus clouds formed by homogeneous freezing
}

\author{
B. Kärcher ${ }^{1,2}$ \\ ${ }^{1}$ Deutsches Zentrum für Luft- und Raumfahrt, Institut für Physik der Atmosphäre, Oberpfaffenhofen, Germany \\ ${ }^{2}$ Ludwig-Maximilians-Universität München, Meteorologisches Institut, München, Germany
}

Received: 21 February 2002 - Published in Atmos. Chem. Phys. Discuss.: 3 April 2002

Revised: 31 May 2002 - Accepted: 3 June 2002 - Published: 24 June 2002

\begin{abstract}
Number concentrations and mean sizes of ice crystals and derived microphysical and optical properties of subvisible cirrus clouds (SVCs) formed by homogeneous freezing of supercooled aerosols are investigated as a function of temperature and updraft speed of adiabatically ascending air parcels. The properties of such clouds are insensitive to variations of the aerosol number and size distribution. Based on criteria constraining the optical extinction, sedimentation time, and existence time of SVCs, longer-lived (> $10 \mathrm{~min}$ ) clouds, capable of exerting a measurable radiative or chemical impact, are generated within a narrow range of updraft speeds below $1-2 \mathrm{~cm} \mathrm{~s}^{-1}$ at temperatures below about $215 \mathrm{~K}$, with concentrations of ice crystals not exceeding $0.1 \mathrm{~cm}^{-3}$. The clouds do not reach an equilibrium state because the ice crystals sediment out of the formation layer typically before the supersaturation is removed. Two important conclusions emerge from this work. First, the above characteristics of SVCs may provide an explanation for why SVCs are more common in the cold tropical than in the warmer midlatitude tropopause region. Second, it seems likely that a limited number $\left(<0.1 \mathrm{~cm}^{-3}\right)$ of effective heterogeneous freezing nuclei that nucleate ice below the homogeneous freezing threshold can control the formation and properties of SVCs, although homogeneous freezing nuclei are far more abundant.
\end{abstract}

\section{Introduction}

Cirrus clouds forming in the tropopause region often stay optically thin and even invisible as appearing to a ground-based observer at most zenith angles (e.g. Lynch, 1993). Frequencies of occurrence and optical extinction of subvisible cirrus (SVC) are routinely measured by the satellite-borne Stratospheric Aerosol and Gas Experiment (SAGE) II (Wang et al.,

Correspondence to: B. Kärcher (bernd.kaercher@ dlr.de)
1996), by the Halogen Occultation Experiment (HALOE) (Hervig and McHugh, 1999), and by lidars. Long-term lidar studies of cirrus including SVCs have recently been reported (Goldfarb et al., 2001; Sassen and Campbell, 2001). Comprehensive in situ studies of SVCs simultaneously using cloud probes and instruments to characterize the interstitial aerosol and cloud particle residues are lacking.

Thin cirrus clouds appear mostly below the local tropopause, especially in the tropics (Beyerle et al., 1998; McFarquhar et al., 2000) and at midlatitudes (Sassen and Campbell, 2001). Sometimes they are also observed above the local tropopause at midlatitudes (Goldfarb et al., 2001) and in polar regions (Lelieveld et al., 1999; Kärcher and Solomon, 1999). As the mean distance between the tropopause and the hygropause increases from $1 \mathrm{~km}$ at low latitudes to $4 \mathrm{~km}$ at high latitudes (Chiou et al., 1997), the probability for SVCs to form above the tropopause increases from the equator to the poles whenever the formation conditions are met. Supersaturation with respect to ice is known to occur in the upper troposphere and lowermost stratosphere (Murphy et al., 1990; Gierens et al., 1999; Ovarlez et al., 2000; Jensen et al., 2001).

Thin and subvisible cirrus prevailing in the tropical upper troposphere play an important role in the dehydration of air entering the tropical lower stratosphere (Jensen et al., 1996). They also control the net radiative heating rates in the tropical lower stratosphere, thereby influencing the abundance of ozone at higher latitudes and the circulation of the stratosphere (Dessler et al., 1996). The chemical effects of high ice clouds on ozone, proposed by Borrmann et al. (1996) to occur at mid and high latitudes via heterogeneous activation of chlorine, remain a subject of debate (Solomon et al., 1997; Smith et al., 2001; Meilinger et al., 2001).

To date, formation mechanisms, properties, and lifetimes of SVCs are only poorly understood. In particular, it is unclear whether SVCs form by homogeneous or by heterogeneous freezing (Kärcher and Solomon, 1999). This is partly 
caused by the fact that the abundance and the freezing characteristics of heterogeneous nuclei that could form ice clouds in the tropopause region are not well known.

While the simulations performed by Jensen et al. (2001) suggest that ice crystals in subvisible cirrus can nucleate heterogeneously, the present work demonstrates that such clouds can also form by homogeneous freezing under suitable atmospheric conditions. Homogeneous freezing can always occur on the ubiquitous liquid sulfate aerosols, but the occurrence of heterogeneous freezing requires solid icenucleating particles (mineral dust, black carbon, partially deliquesced ammoniated sulfates) the abundance of which in the upper troposphere may show significant seasonal and geographic variances.

While few numerical simulations of the formation and evolution of SVCs are available, a survey of the properties of SVCs (number and mean sizes of ice crystals and derived microphysical and optical properties) as a function of the variables that control the formation of such clouds (updraft speed, temperature, freezing behavior of aerosols) has not yet been performed. I employ purely analytic means to achieve this goal in the present work. It is the simplicity of the modeling and the generality of the approach that renders this type of study a viable complement to numerical cloud models.

I focus on SVCs formed by homogeneous freezing of supercooled aerosol particles, for which the freezing relative humidities have been determined in the laboratory for a variety of particle types relevant to ice formation in the atmosphere. Further, I concentrate on clouds generated in situ by adiabatic cooling of air parcels, a very common process leading to ice supersaturation in the atmosphere. Adiabatic cooling can be driven by the mean circulation (Hadley cell), by planetary (Kelvin) waves, by synoptic-scale (baroclinic) instabilities, by gravity waves, or by convection. Occasionally, thin cirrus shields can be formed in the outflow of glaciating cumulus anvils followed by horizontal spreading, by isobaric mixing of air parcels with different temperature and humidity, or by radiative cooling. Such forcings are not covered by the present approach.

The objective of Sect. 2 is to briefly recall the theoretical basis which I have developed in previous studies. I refer the reader to the original references for a more detailed justification of the approach. In Sect. 3 I present a detailed investigation of the key properties of SVCs. In Sect. 4 I summarize the results and address the implications concerning the formation mechanisms of SVCs. In Sect. 5 I list several mathematical symbols repeatedly used throughout this work.

\section{Theory}

\subsection{Homogeneous freezing thresholds}

I apply the homogeneous freezing formulation for chemically uniform, liquid aerosols developed by Koop et al. (2000) from a series of laboratory studies. Let $S$ denote the saturation ratio with respect to ice, defined as the ratio of the number density of $\mathrm{H}_{2} \mathrm{O}$ molecules in the gas phase and the $\mathrm{H}_{2} \mathrm{O}$ saturation number density $e(T)$ over ice. The dependences of the ice saturation ratio on temperature $T$ at the observed freezing thresholds $S_{\mathrm{cr}}$ were found to be independent of the chemical nature of the solutes.

In this work I use the numerical fit

$S_{\text {cr }}=2.583-T[\mathrm{~K}] / 207.83$,

derived from the model parameterization for the measured freezing rates provided by Koop et al. (2000). Values for $S_{\mathrm{cr}}$ range from about 1.4 to 1.7 for $T$ between $235 \mathrm{~K}$ and $195 \mathrm{~K}$, corresponding to supercoolings of $\sim 3-3.5 \mathrm{~K}$.

Ice particles form and grow above $S_{\text {cr }}$. Hereafter the saturation with respect to ice $S$ reaches a maximum value $S_{\max } \gtrsim S_{\text {cr }}$ and falls off rapidly because the growing ice particles deplete the available water vapor. It is important to note that freezing of solution droplets only takes place in a narrow range between $S_{\mathrm{cr}}$ and $S_{\mathrm{max}}$.

\subsection{Ice particle concentrations}

The number density of ice crystals $n$ formed during the freezing event and the radius $r_{s}$ of the smallest aerosol particle that freezes are obtained from the following set of equations (Kärcher and Lohmann, 2002a,b):

$$
\begin{aligned}
& n=\int_{r_{s}}^{\infty} d r_{0} \frac{d n_{a}}{d r_{0}} \leq n_{a}, \\
& \frac{a_{1} S_{\mathrm{cr}}}{a_{2}+a_{3} S_{\mathrm{cr}}} w=\int_{r_{s}}^{\infty} d r_{0} \frac{d n_{a}}{d r_{0}} \mathcal{R}, \\
& \mathcal{R}=\frac{4 \pi}{v} \frac{b_{1}}{b_{2}^{2}} \frac{\delta^{2}}{1+\delta}\left\{1-\frac{1}{\delta^{2}}+\right. \\
& \left.\frac{1}{\delta^{2}}\left[\frac{(1+\delta)^{2}}{2} \sqrt{\kappa}+\frac{1}{\sqrt{\kappa}}\right] \sqrt{\pi} \exp \left(\frac{1}{\kappa}\right) \operatorname{erfc}\left(\frac{1}{\sqrt{\kappa}}\right)\right\} .
\end{aligned}
$$

In these equations, $n_{a}$ is the total number of aerosol particles, $d n_{a} / d r_{0}$ is the aerosol particle size distribution, $w$ is the updraft speed of the air parcel assumed to be constant, $v$ is the specific volume of a water molecule in ice, and $\operatorname{erfc}(x)=1-(2 / \sqrt{\pi}) \int_{0}^{x} d t \exp \left(-t^{2}\right)$.

The coefficients $a_{k}$ and $b_{k}$ depend on $T$ and air pressure $p$ and are determined by the adiabatic changes of the state variables and the ice particle growth law. In particular, $a_{1}=L_{s} M_{w} g /\left(c_{p} R T^{2}\right)-M g /(R T)$, with the molecular masses of air $M$ and water $M_{w}$, latent heat of sublimation $L_{s}$, constant of gravity $g$, heat capacity at constant pressure $c_{p}$, and the universal gas constant $R ; a_{2}=1 / e ; a_{3}=$ 
$L_{s}^{2} M_{w} m_{w} /\left(c_{p} p T M\right)$, with the mass of a water molecule $m_{w}$. Further, $b_{1}=v e(S-1) \alpha \bar{v} / 4$ and $b_{2}=\alpha \bar{v} /\left(4 D_{*}\right)$, with the deposition coefficient $\alpha$ of $\mathrm{H}_{2} \mathrm{O}$ molecules on ice, their thermal speed $\bar{v}$, and their diffusion coefficient $D_{*}(T, p)$ in air. Finally the parameters $\delta$ and $\kappa$ are defined by:

$\delta=b_{2} r_{0}, \quad \kappa=2 b_{1} b_{2} \tau(1+\delta)^{-2}$,

where $\tau \propto 1 / w$ is the characteristic timescale of the freezing event. At $\delta \approx 1$, gas kinetic and diffusive effects on the ice particle growth rate are of similar importance. At $\kappa \approx 1$, freezing of aerosol particles and subsequent growth of the ice particles in the formation stage occur over similar timescales. Ice crystal sedimentation out of the air parcel is not included in the above equations. Fallout and freezing timescales are usually well separated so that sedimentation will not significantly alter the number of ice particles.

The mean size $\hat{r}$ of the ice particles at the end of the initial growth phase, that is, when $S$ starts falling below $S_{\text {cr }}$ and freezing is terminated, is computed from

$$
\frac{1+b_{2} \hat{r}}{1+b_{2} r_{s}}=1+\frac{\sqrt{\pi \kappa}}{2} \exp \left(\frac{1}{\kappa}\right) \operatorname{erfc}\left(\frac{1}{\sqrt{\kappa}}\right) \text {. }
$$

Since depositional growth of ice particles continues after the formation stage, Eq. (6) generally underestimates their mean size (and mass). An approximate analytical method to take this later growth stage into account as a function of time until equilibrium is approached $(S \rightarrow 1)$ is outlined in Sect. 2.3.

My present choice of the deposition coefficient, $\alpha=0.5$, is motivated by the experimental studies of Haynes et al. (1992). These authors accurately measured deposition and evaporation coefficients of $\mathrm{H}_{2} \mathrm{O}$ molecules on ice surfaces at low temperatures $(<205 \mathrm{~K})$ using optical interference techniques. This value is roughly consistent with the results from two field measurements of cirrus clouds (Kärcher and Lohmann, 2002a). In a cirrus model intercomparison exercise, it was found that the predicted number of ice crystals was relatively insensitive to changes of $\alpha$ in the regime $0.2 \leq \alpha \leq 1$ (Lin et al., 2002).

\subsection{Ice particle sizes}

The approximate temporal evolution of the size of small, spherical ice crystals that grow in an ice-supersaturated environment can be obtained in closed form by considering the $\mathrm{H}_{2} \mathrm{O}$ mass balance and the radial growth rate of the particles (Kärcher and Solomon, 1999).

The overall $\mathrm{H}_{2} \mathrm{O}$ mass balance reads $m_{\infty}=m_{0}+m_{w} e s_{0}$, in terms of the ice water mass $m$ and with the supersaturation $s=S-1$. The subscripts 0 and $\infty$ denote initial (at $S=S_{\text {cr }}$ as defined in Sect.2.1) and final (at $S=1$ ) values, respectively. The ice particle number density, temperature, and pressure are assumed to stay constant during growth and the updraft ceases shortly after $S$ reaches its peak value.

From the conservation of $\mathrm{H}_{2} \mathrm{O}$ mass it follows

$s(r)=\frac{4 \pi n}{3 v e}\left(r_{\infty}^{3}-r^{3}\right)$ and the final ice particle radius after the supersaturation relaxes to zero is given by

$r_{\infty}=\left(r_{0}^{3}+\frac{3 v e}{4 \pi n} s_{0}\right)^{1 / 3}$.

The initial radius of the ice particles is given by $r_{0}=\hat{r}$ as defined in Eq. (6). Consistent with the equations given in Sect. 2.2, the approximate ice particle growth law reads

$\frac{d r}{d t}=\frac{b_{1}}{1+b_{2} r}=v e \frac{D(r) s(r)}{r}$,

where the effective gas diffusion coefficient $D$ for $\mathrm{H}_{2} \mathrm{O}$ molecules in air is given by

$D(r)=D_{*}\left(1+\frac{4 D_{*}}{\alpha \bar{v} r}\right)^{-1}$,

with the uncorrected diffusion coefficent $D_{*}$.

Defining $x=r / r_{\infty}$ and $\tau_{g}=t / t_{g}$, with the growth timescale $t_{g}$ characteristic for the relaxation phase given by

$t_{g}=3\left(4 \pi n D_{*} r_{\infty}\right)^{-1}$;

substituting $s$ from Eq. (7) and $D$ from Eq. (10) into Eq. (9) yields

$\tau_{g}(x)=\int_{x_{0}}^{x} d x \frac{x(1+\beta / x)}{1-x^{3}}, \quad \beta=\frac{1}{b_{2} r_{\infty}}=\frac{4 D_{*}}{\alpha \bar{v} r_{\infty}}$.

The scaled form of the integral reveals that the solutions only depend on the dimensionless parameter $\beta$ and on $x_{0}$. With the functions

$\mathcal{I}_{1}(x)=\frac{1}{6} \ln \left[\frac{1+x+x^{2}}{(1-x)^{2}}\right]$,
$\mathcal{I}_{2}(x)=\frac{1}{\sqrt{3}} \arctan \left(\frac{1+2 x}{\sqrt{3}}\right)$,

the result reads

$$
\begin{aligned}
\tau_{g}(x) & =(\beta+1)\left[\mathcal{I}_{1}(x)-\mathcal{I}_{1}\left(x_{0}\right)\right] \\
& +(\beta-1)\left[\mathcal{I}_{2}(x)-\mathcal{I}_{2}\left(x_{0}\right)\right] .
\end{aligned}
$$

Equation (15) is a convenient expression to compute the growth history $r(t)$ of the ice particles for a given number density and initial radius and supersaturation after conversion to physical units.

The analytic model assumes that the updraft ceases shortly after the saturation reaches its peak value. In a real air parcel, cooling may continue after most of the ice particles have formed. While this approximation does not affect the prediction of $n$ (as the pristine ice particles typically remove supersaturation faster than it is produced by a continuing uplift), the ice particle radii $r>\hat{r}$ may be underestimated when ongoing cooling produces a small (compared to $S_{\mathrm{cr}}-1$ ) residual supersaturation.

However, this effect is relatively small under the conditions relevant for the formation of SVCs (low updraft speeds 

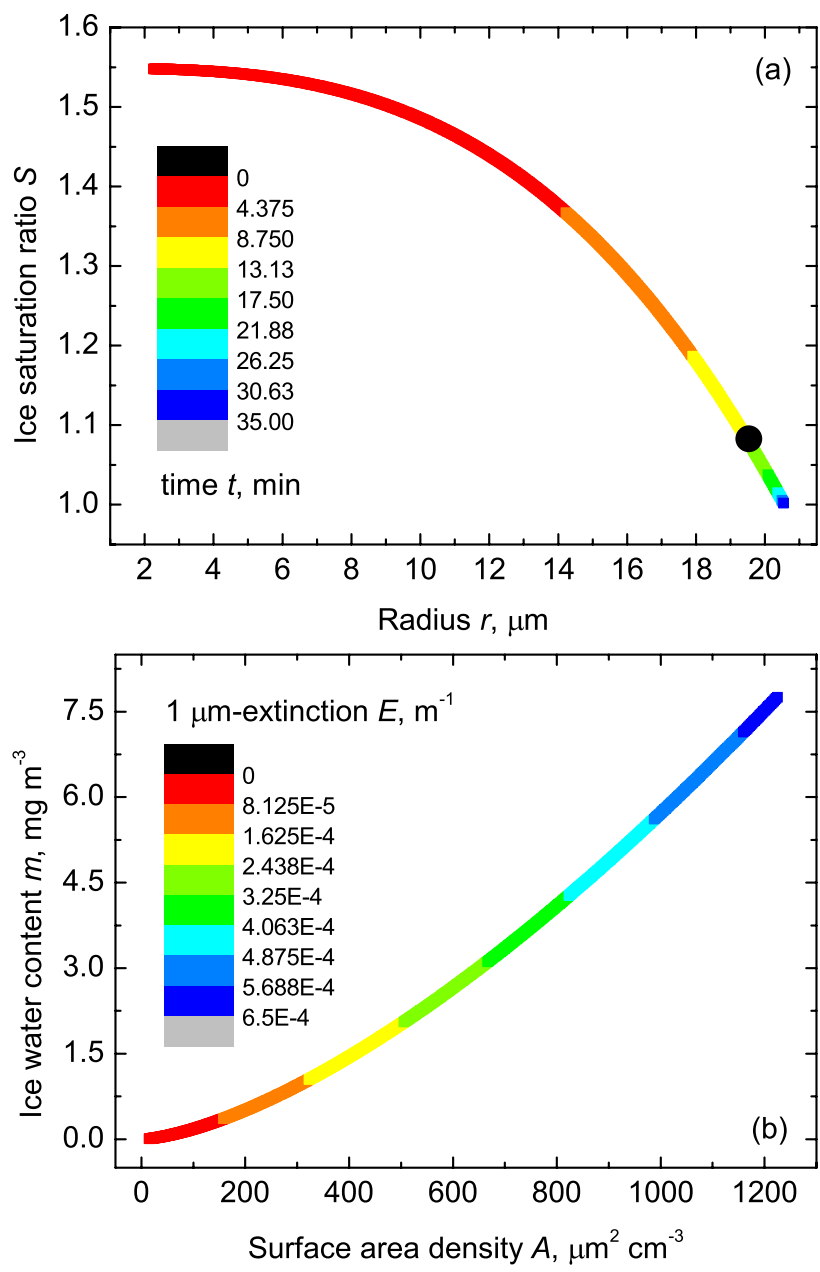

Fig. 1. Approximate evolution of a cirrus cloud in the tropopause region after the formation stage. (a) Ice saturation ratio versus mean ice particle radius. The color coding indicates the time elapsed during the growth towards equilibrium. The black circle locates the value of the characteristic relaxation time $t_{g}$ during which the cloud volume remains substantially supersaturated with respect to ice. (b) Ice water content versus specific cloud surface area. The color coding indicates the cloud extinction at a wavelength of $1 \mu \mathrm{m}$. For details of the initialization, see text.

and low temperatures); see Sect. 3.5. To give one example from a parcel model simulation conducted at $w=1 \mathrm{~cm} \mathrm{~s}^{-1}$ for a freezing temperature of $209 \mathrm{~K}$, I deduce a mean ice particle size $r$ of $46 \mu \mathrm{m}$ when cooling stops after freezing and a value $r=50 \mu \mathrm{m}$ with ongoing cooling 4 hours after the ice particles were formed (in this case the residual supersaturation is $3 \%$ and $n=0.008 \mathrm{~cm}^{-3}$ ).

\subsection{Optical extinction}

I determine the optical extinction $E$ of the cirrus cloud at a given wavelength $\lambda$, assuming a monodisperse ice crystal spectrum. With the extinction efficiency $Q(r, \lambda, \mu)$ taken from Mie theory, this coefficient is defined as

$E=\pi r^{2} Q n=Q A / 4, \quad A=4 \pi r^{2} n$,

where I have introduced the real refractive index $\mu$ for pure ice $(\mu=1.31)$ and the specific surface area density $A$ of the cloud particles. For the Mie extinction efficiency, I choose the approximate expression

$Q=2-\frac{4}{\varrho}\left[\sin (\varrho)-\frac{1-\cos (\varrho)}{\varrho}\right], \varrho=\frac{4 \pi r(\mu-1)}{\lambda}$

derived by van de Hulst (1957). This formula is valid for $1>\mu>2$ and approximately describes the salient features of the Mie extinction curve. It is not applicable in the Rayleigh scattering regime and underestimates the exact extinction values by less than $20 \%$ in the Mie and geometrical regimes.

In terms of extinction as measured by SAGE II, cirrus clouds may be classified as subvisible when the following criteria are met (Kent et al., 1993):

$$
\begin{aligned}
& E(\lambda=1 \mu \mathrm{m})>(5-8) \times 10^{-7} \mathrm{~m}^{-1}, \\
& E(\lambda=1 \mu \mathrm{m})<(2-3) \times 10^{-5} \mathrm{~m}^{-1}, \\
& \frac{E(\lambda=0.5 \mu \mathrm{m})}{E(\lambda=1 \mu \mathrm{m})}<2 .
\end{aligned}
$$

The boundary between aerosol and SVC is given by Eq. (18), and that between SVC and opaque clouds by Eq. (19). For $r>1 \mu \mathrm{m}$, Eq. (20) is always fulfilled; see Fig. A1 in Kärcher and Solomon (1999). As most ice crystals are larger than 1 $\mu \mathrm{m}$, the last criterion does not need to be considered further for the purposes of this work.

The above classification of SVCs roughly corresponds to visible optical depths below about $0.03-0.05$, a visibility threshold frequently used in lidar studies (Sassen and Cho, 1992). I prefer to discuss cloud extinction rather than optical depth because cirrus clouds exhibit a broad range of geometrical thicknesses.

\section{Results and discussion}

\subsection{General cloud features}

The ice particle number density $n$ is calculated at a given freezing temperature, updraft speed, and aerosol particle size distribution using the parameterization scheme summarized in Sect. 2.1 and 2.2. Throughout this work, the air pressure is fixed to a value of $180 \mathrm{hPa}$ at the point of cloud formation, as the results are not sensitive to this value. In the formation stage, the ice saturation obtains its approximate peak value of $S_{\mathrm{cr}}$ determined by the homogeneous freezing process. At the end of the formation stage, the monodisperse ice particles have grown to a radius of $\hat{r}$.

As outlined in Sect. 2.3, the subsequent growth stage is characterized by the relaxation of $S$ from $S_{\text {cr }}$ to its equilibrium value of unity; it is assumed that the air parcel stops 
rising after the freezing event (temperature and ice number densities are kept constant) and no further ice nucleation takes place. The final size of the ice particles at the end of the relaxation stage is determined by the supersaturation $\left(S_{\text {cr }}-1\right)$. The volume extinction $E$ of the cloud particles at visible wavelengths is determined using the simplified model described in Sect. 2.4.

Figure 1 shows the time history of the key properties of a thin cirrus cloud. The cloud formed at a freezing temperature of $215 \mathrm{~K}$ in an air parcel initially ascending with $w=10 \mathrm{~cm} \mathrm{~s}^{-1}$. The prescribed aerosol size distribution is lognormal, with $n_{a}=200 \mathrm{~cm}^{-3}$, a mean number radius of $45 \mathrm{~nm}$ (dry radius $20 \mathrm{~nm}$ ), and a variance of 1.8. These parameters have been used as baseline values in the cirrus parcel model intercomparison project conducted within the Global Energy and Water Cycle Experiment (GEWEX), see Lin et al. (2002). The resulting number of ice particles is $n=0.23 \mathrm{~cm}^{-3}$. I will use the above spectral parameters in all subsequent calculations, as the sensitivity of $n$ to details of the size spectrum is small; see Sects. 3.3 and 3.4.

As the saturation ratio decreases from its approximate peak value of 1.55 to unity, the mean size of the ice particles increases from its initial value of $2.25 \mu \mathrm{m}$ to its final value of $20.6 \mu \mathrm{m}$ (Fig. 1a). The characteristic timescale $t_{g}$ from Eq. (11) is about 13 min (see black circle), and equilibrium is practically reached after about $30 \mathrm{~min}$. The initial ice water content of $0.01 \mathrm{mg} \mathrm{m}^{-3}$ and surface area density of $14.8 \mu \mathrm{m}^{2} \mathrm{~cm}^{-3}$ increase to their final values $7.8 \mathrm{mg} \mathrm{m}^{-3}$ and $1225 \mu \mathrm{m}^{2} \mathrm{~cm}^{-3}$, respectively (Fig. 1b). Consistent with these changes, the $1 \mu \mathrm{m}$-extinction rises from $6.7 \times 10^{-6}$ $\mathrm{m}^{-1}$ to $6.3 \times 10^{-4} \mathrm{~m}^{-1}$, that is, from a value below to a value above the visibility threshold Eq. (19).

After about $t_{v}=20 \mathrm{~s}$, the particles achieve a mean radius of $4.3 \mu \mathrm{m}$ (almost twice the initial radius, not visible in Fig. 1a), indicating rapid deposition of $\mathrm{H}_{2} \mathrm{O}$ molecules in the early growth stage of the relaxation phase where the clouds exhibit the lowest extinction values. At this point, the extinction threshold Eq. (19) is surpassed and the cloud becomes visible.

One may expect that lowering the value of the deposition coefficient $\alpha$ would extend the duration of this subvisible stage because the growth rates become smaller. However, lower values of $\alpha$ allow more aerosol particles to freeze as the first freshly produced ice crystals are less effective in depleting the available $\mathrm{H}_{2} \mathrm{O}$. This leads to a significant decrease of the overall growth timescale $t_{g}$ and thus of $t_{v}$.

Using $\alpha=0.2$ instead of 0.5 , the number of ice crystals formed increases from $0.23 \mathrm{~cm}^{-3}$ to $0.52 \mathrm{~cm}^{-3}, t_{g}$ decreases from $13 \mathrm{~min}$ to $7.3 \mathrm{~min}$, and it takes only $10 \mathrm{~s}$ until the cloud becomes visible. Using $\alpha=0.05, n=6.6 \mathrm{~cm}^{-3}, t_{g}=$ $1.3 \mathrm{~min}$, and $t_{v}=2 \mathrm{~s}$. In summary, the uncertainty in $t_{v}$ associated with changes of $\alpha$ is of the order of a factor $2-$ 10 , with relatively small sensitivities for values $\alpha>0.2$. However, at the low temperatures considered here, the use of

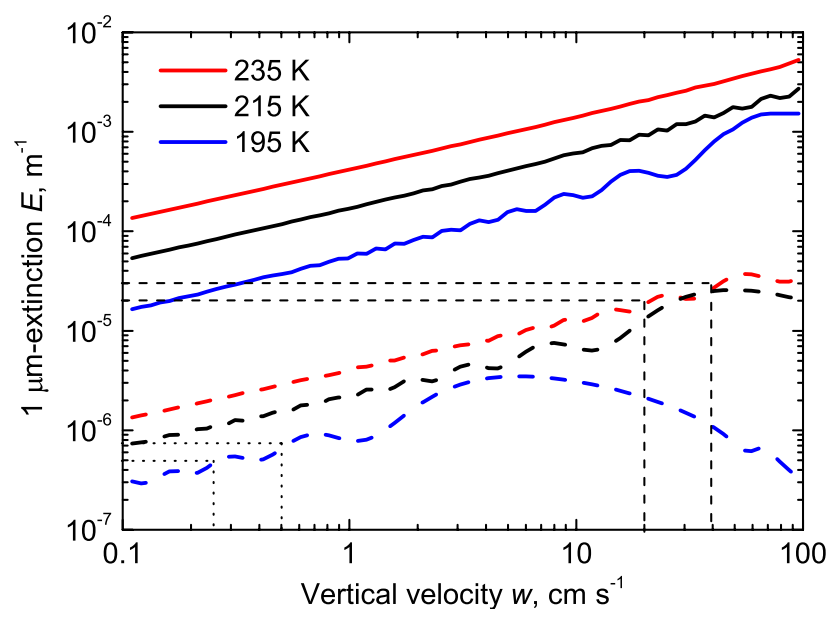

Fig. 2. Volume extinction of monodisperse ice particles at $1 \mu \mathrm{m}$ wavelength $\lambda$ of developing thin cirrus clouds versus vertical velocity for different freezing temperatures (see legend) at the time of cloud formation ( $S=S_{\mathrm{cr}}$, lower dashed curves) and at equilibrium ( $S=1$, upper solid curves). Extinction rises from the former initial values to the latter final values, assuming constant temperature and ice particle concentration, and provided that the updraft ceases shortly after formation. The Mie oscillations are visible for ice particles with sizes of the order $\lambda$. The dotted/dashed lines bracket the ranges of the extinction threshold below which the SAGE II sensors detect aerosols/subvisible clouds.

values $\alpha<0.2$ is not supported by laboratory measurements (Haynes et al., 1992).

\subsection{Cloud extinction}

To determine under which formation conditions the cirrus clouds are subvisible, I discuss the cloud extinction $E$ over a wide range of updraft speeds $w$ and temperatures $T$. Figure 2 displays the extinction at $\lambda=1 \mu \mathrm{m}$ versus vertical velocity for different freezing temperatures. Generally, the cloud extinction decreases with decreasing $T$ and $w$.

At the end of the formation stage (lower dashed curves), most cloud extinction values lie above the range of SAGE II threshold values between aerosols and SVCs (dotted lines) given by Eq. (18), except those for the cold case (195 K, blue dashed curve) for $w<0.25-0.5 \mathrm{~cm} \mathrm{~s}^{-1}$. The range of SAGE II cloud visibility threshold values from Eq. (19) (dashed lines) shows that initially the most of the clouds are subvisible, except those for the warmer cases $(215 \mathrm{~K}$ and $235 \mathrm{~K}$ ) for $w>20-40 \mathrm{~cm} \mathrm{~s}^{-1}$. Since all final extinction values (upper solid curves) lie above the visibility threshold, the clouds become visible at some point during their growth phase, as discussed in Sect.3.1. I return to this issue in Sect. 3.5. To further interpret the results shown in Fig. 2, I examine the concentrations (number, surface area, and mass) and sizes of the cloud particles next. 


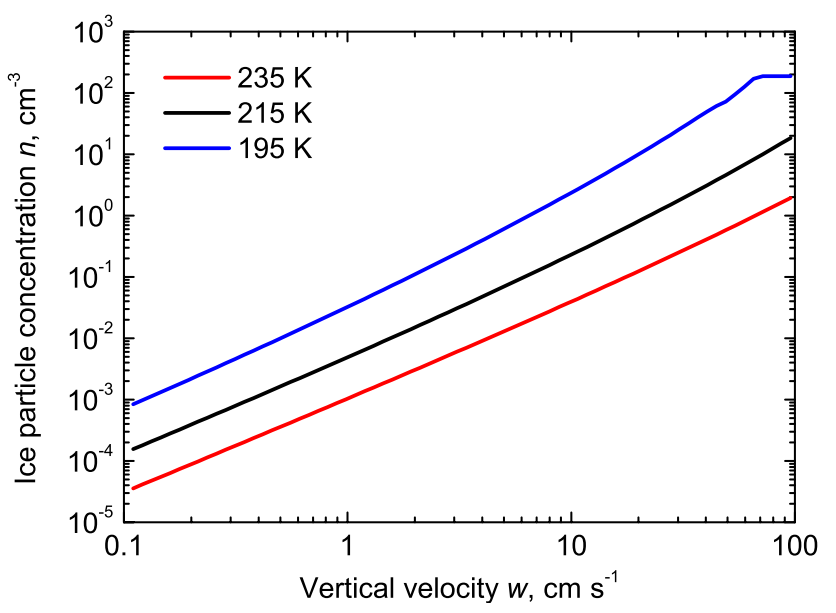

Fig. 3. Number density of ice particles as a function of the vertical velocity for three freezing temperatures. In the $195 \mathrm{~K}$-case, $n$ is limited by the total number of aerosol particles available for freezing for $w>70 \mathrm{~cm} \mathrm{~s}^{-1}$.

\subsection{Cloud particle concentrations and sizes}

The number densities of ice particles as a function of vertical velocity and freezing temperature are presented in Fig. 3 . The ice particle concentrations $n$ increase strongly with rising $w$ (increased dynamic forcing) and decrease with rising $T$ (increased growth rates), as discussed by Kärcher and Lohmann (2002a). The curves $n(w)$ are mostly straight lines on the double-logarithmic scale. Slight deviations from straight lines occur at rather high vertical velocities $(w>10$ $\mathrm{cm} \mathrm{s}^{-1}$ ), especially at low temperatures. These deviations are mainly caused by aerosol size effects (Kärcher and Lohmann, 2002b). In addition, the blue curve in Fig. 3 indicates that $n$ can become limited by the total number of aerosol particles available for freezing (here $n_{a}=200 \mathrm{~cm}^{-3}$ ) in strong updrafts.

Figure 4 depicts the corresponding behavior of mean ice particle radius (a), total cloud surface area density (b), and ice water content (c). As in Fig. 2, the dashed curves are taken right after cloud formation is completed and the solid curves represent the final equilbrium state at the end of the relaxation phase. While $r$ decreases with rising $w, A$ increases. Ice water contents are almost independent of $w$.

\subsection{Scaling properties}

The fact that SVCs preferentially form in moderate updrafts and exist over extended periods of time only at relatively low temperatures allows simple scaling laws to be applied to describe the dependence of the microphysical properties of SVCs on $w$ and T. The straight curves in Fig. 3 and Fig. 4 already suggest that $n, r$, and the derived quantities can be approximated by power laws in terms of $w$.
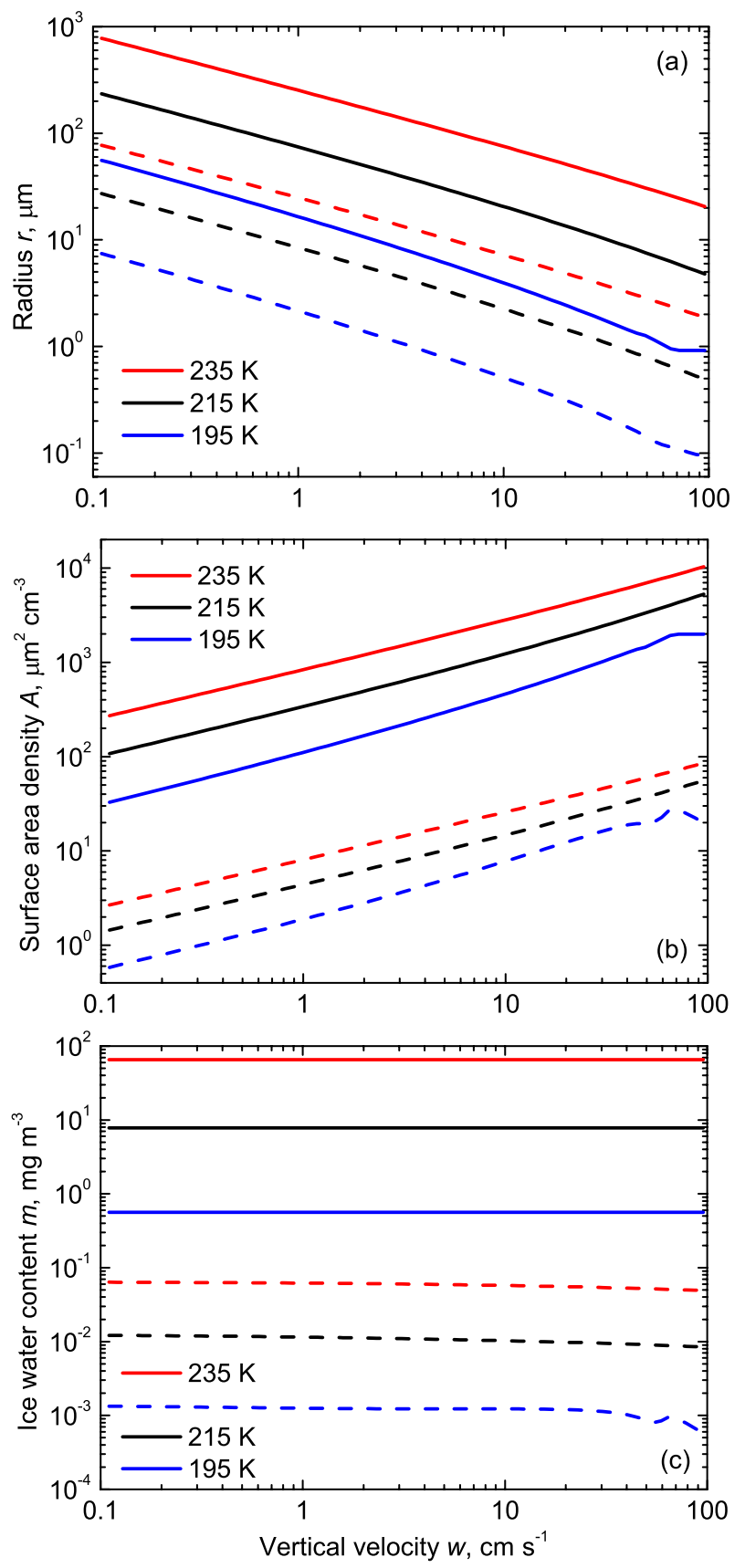

Fig. 4. Mean radius (a), surface area density (b), and water content (c) of ice particles as a function of the vertical velocity for three freezing temperatures (see legend). The corresponding number densities of the cloud particles are shown in Fig. 3. Dashed curves are taken at the time of cloud formation, solid curves represent values after the relaxation phase at equilibrium.

As explained by Kärcher and Lohmann (2002a,b), cirrus formation is very insensitive to the number and size of aerosol particles present in weak updrafts $\left(w<10 \mathrm{~cm} \mathrm{~s}^{-1}\right)$, provided that homogeneous freezing is the only path to ice formation. In this limit, the microphysical properties solely 


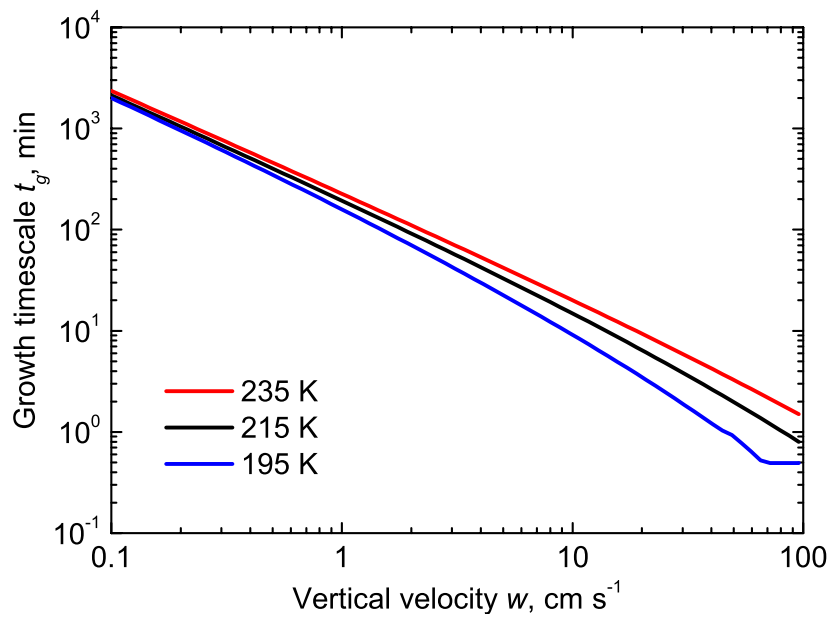

Fig. 5. Growth timescales in the relaxation phase as a function of the vertical velocity for three freezing temperatures. Within this time span, the cloud volume remains supersaturated, thermodynamic equilibrium is approached, and ice particle growth ceases. The values $t_{g}$ mark the approximate time (within a factor of two) evolving between the dashed curves (just after formation of SVCs) and the solid curves (when the supersaturation vanishes) in Fig. 2 and Fig. 4; recall the discussion in Sect. 3.1.

depend on $w$ and $T$ (the latter mostly via the ice saturation vapor pressure $e$ ). The scaling laws for $n$ and $\hat{r}$ read (Kärcher and Lohmann, 2002a),

$n \propto w^{3 / 2} e^{-1 / 2}$,

$\hat{r} \propto w^{-1 / 2} e^{1 / 2}$.

From these relationships, one easily verifies that

$\hat{A} \propto n^{1} \hat{r}^{2} \propto w^{1 / 2} e^{1 / 2}$,

$\hat{m} \propto n^{1} \hat{r}^{3} \propto w^{0} e^{1}$.

Inspection of Fig. 4a shows that $\hat{r}$ is about an order of magnitude smaller than $r_{\infty}$, that is $\hat{r} \ll r_{\infty}$. Therefore, with the help of Eq. (8), the scaling laws for $r_{\infty}, A_{\infty}$, and $m_{\infty}$ read

$r_{\infty} \propto n^{-1 / 3} e^{1 / 3} \propto w^{-1 / 2} e^{1 / 2}$,

$A_{\infty} \propto n^{1} r_{\infty}^{2} \propto w^{1 / 2} e^{1 / 2}$,

$m_{\infty} \propto n^{1} r_{\infty}^{3} \propto w^{0} e^{1}$,

identical to Eqs. (22)-(24).

For the growth timescale Eq. (11), the scaling reads

$t_{g} \propto n^{-1} r_{\infty}^{-1} \propto w^{-1} e^{0}$.

Values of $t_{g}$ are depicted in Fig. 5. Recall that during $t_{g}$, the cloud volume is supersaturated with respect to ice; see Fig. 1a. The scaling law Eq. (28) indicates that the times needed to reach equilibrium are only weak functions of $T$ but increase strongly with decreasing $w$. The cloud evolution will be interrupted if changing environmental conditions remove the supersaturation faster than $t_{g}$ or if ice particles

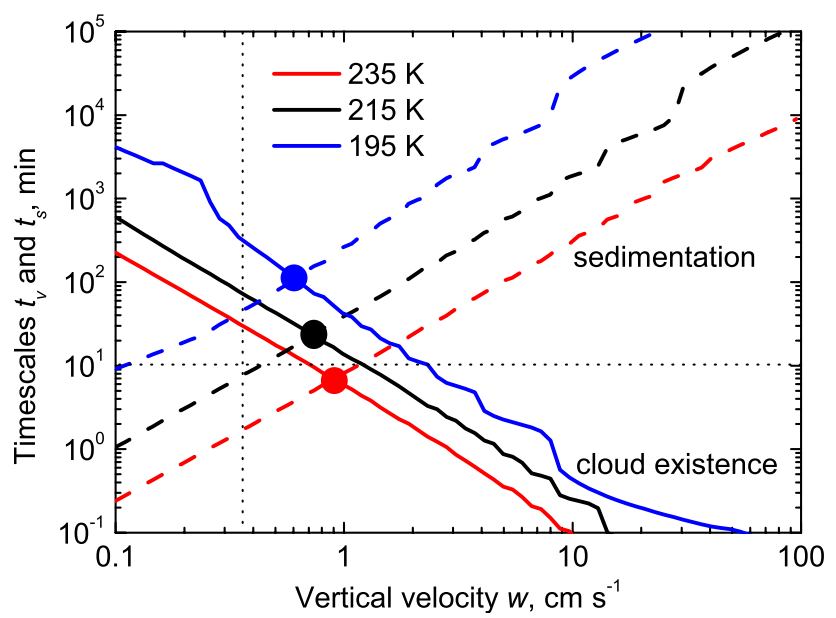

Fig. 6. Time spans from the point of subvisible cloud formation to the point where the clouds become visible (solid curves) and lowerlimit timescales for sedimentation of ice particles out of a layer of $750 \mathrm{~m}$ thickness (dashed curves) versus vertical velocity for three freezing temperatures. The colored circles mark the values of $w$ for which both timescales are equal for each $T$. The vertical dotted line marks the approximate lower limit for $w$ arising from Eq. (18); see also Fig. 2. Below the horizontal dotted line SVCs are only shortlived transient phenomena with little atmospheric significance.

sediment out of the cloud layer faster than $t_{g}$. This is explored in more detail in Sect. 3.5.

I further note an approximate scaling law for the sedimentation velocity $v_{s}$ of spherical particles of radius $\hat{r}<r<r_{\infty}$. As outlined in the next section, $v_{s}$ is proportional to $r^{2}$, apart from slip-flow corrections and form factors. Particles fall out of a cloud layer of vertical thickness $\Delta z$ within the characteristic timescale $t_{s}=\Delta z / v_{s}$. The corresponding scaling law for $t_{s}$ reads

$t_{s} \propto n^{0} r^{-2} \propto w^{1} e^{-1}$,

as both $\hat{r}$ and $r_{\infty}$ exhibit the same dependences on $w$ and $e$. Reported mean geometrical thicknesses $\Delta z$ of thin and subvisible cirrus are $470 \mathrm{~m}$ for tropical clouds (Winker et al., 1998) and $930 \mathrm{~m}$ in cloud samples observed at midlatitudes (Sassen and Cho, 1992).

Deviations from the above scaling laws, as noted by careful inspection of Figs. 3-5, arise from aerosol size effects (Kärcher and Lohmann, 2002b) and to a lesser degree from dependences on $T$ that are not captured by the exponential dependence via $e(T)$, i.e., through the parameters $D_{*}$ and $\bar{v}$.

\subsection{Subvisible cloud existence times}

I have computed the time spans $t_{v}$ from the point of subvisible cloud formation to the point where the clouds become visible, plotted as solid curves in Fig. 6, with the help of the extinction threshold criteria Eq. (18) and Eq. (19). To estimate the sedimentation timescale $t_{s}$ (dashed curves), I have 
used $\Delta z=750 \mathrm{~m}$ and the fall speed $v_{s}=4 \times 10^{6} \mathrm{~cm}^{-1} \mathrm{~s}^{-1} r^{2}$ (Pruppacher and Klett, 1997). This form for $v_{s}$ is strictly valid only for spherical, micrometer-sized particles. The possible asphericity of ice crystals affects $v_{s}$, but the differences (part of which can be compensated by concomitant changes of the growth law) are not relevant for the present discussion. I have evaluated $v_{s}(r)$ using the sizes the ice particles obtained at the point the clouds become visible, hence the timescales $t_{s}$ are lower limits to the actual values. On the other hand, ongoing cooling after freezing may lead to ice particle sizes larger than those used here; this effect is ignored (see Sect. 2.3) but would yield shorter timescales.

For each temperature, the circles in Fig. 6 mark the vertical velocities for which $t_{v}=t_{s}$. To the left of these circles, the cloud existence times increase to significant values $\left(t_{v} \gtrsim 10\right.$ min, dotted horizontal line), but sedimentation is rapidly limiting the SVC lifetime by transport of ice water to lower altitudes. To the right of these circles, the time spans for clouds to stay subvisible quickly become insignificant.

From this I conclude that SVCs are short-lived and hardly observable at temperatures above about $215 \mathrm{~K}$ for all conceivable values of $w$. Below $215 \mathrm{~K}$, SVCs can persist for more than $10 \mathrm{~min}$, but only in a relatively narrow range of $w$ below $1-2 \mathrm{~cm} \mathrm{~s}^{-1}$. The exact lower limit of $w$ at each $T$ is determined both, by the lower extinction limit for SVCs from Eq. (18) (dotted vertical line in Fig. 6) and by the sedimentation timescale $t_{s}$. As $t_{s}$ is approximately equal to or smaller than $t_{g}$ below this limit (compare with Fig. 5), the SVCs do not reach an equilibrium state during their existence times, so that the cloud volume may stay supersaturated with respect to ice.

One may argue that sedimentation may not necessarily limit the lifetime of SVCs when the altitude region in which freezing occurs (in the top region of the cloud) is much smaller than the total cloud thickness and a large fraction of the middle and bottom region of the cloud is attributed to sedimenting ice particles. However, the separation of nucleation and sedimentation layer may not be critical for SVCs, which are vertically very thin and exhibit much less vertical variability in cloud properties as known from thicker cirrus.

Also, SVCs could form from residuals of visible clouds that become subvisible after the large crystals fall out and sublimate, leaving behind only the smaller crystals. A study of the properties of this class of SVCs would require the use of a cloud-resolving model with size-resolved microphysics, and the present results obtained with the analytical model cannot be applied to such SVCs.

\section{Summary and implications}

By applying an analytical model for the homogeneous freezing and growth of atmospheric ice particles, I have shown that the average microphysical properties of SVCs can be described by simple scaling laws connecting cloud particle number and size with the updraft speed and the freezing temperature. The properties of such clouds are insensitive to details of the aerosol size distribution. The analytical results should prove robust, as they are broadly consistent with results from numerical simulations. However, details such as effects occurring from radiative cooling of cirrus in the presence of underlying convective cloud systems (Hartmann et al., 2001) or from a coupling between crystal growth and sedimentation (Peter et al., 2000), both of which can prolong the lifetime of thin tropical SVCs, are not described.

While SVCs originating from homogeneous freezing of supercooled aerosols can form over a wide range of updraft speeds and temperatures, longer-lived (> $10 \mathrm{~min}$ ) SVCs, observable by satellite sensors or in situ measurements and capable of exerting a measureable radiative or chemical impact, will only be generated within a narrow range below $1-2$ $\mathrm{cm} \mathrm{s}^{-1}$ at temperatures below about $215 \mathrm{~K}$. These numbers are based on criteria constraining the extinction, sedimentation time, and existence time of SVCs. The number of crystals formed under such conditions does not exceed $0.1 \mathrm{~cm}^{-3}$. These crystals fall out of the formation layer typically before the supersaturation is removed. This prevents such clouds from reaching an equilibrium state. These findings may provide one explanation for why SVCs are more common in the cold tropical tropopause region than in the relatively warmer midlatitude tropopause region.

The above findings - SVCs form at low temperatures in weak updrafts - agree qualitatively with the numerical simulations presented by Jensen et al. (2001). In these simulations, however, SVCs were assumed to form heterogeneously from a prescribed number of freezing nuclei $\left(\leq 0.01 \mathrm{~cm}^{-3}\right)$ that trigger ice formation at $S_{\mathrm{cr}}=1.33$, well below the homogeneous threshold. Given the narrow range of moderate updraft speeds and the low number densities of crystals formed, the question arises whether heterogeneous freezing nuclei are capable of altering the present results.

Measuring the ice-nucleating ability of aerosol particles in the upper troposphere and lowermost stratosphere is very difficult, and only little is known about the regional and seasonal variation of efficient freezing nuclei. The appearance of high concentrations of such particles may be of episodic nature, tied to convective or frontal systems that transport aerosols from the boundary layer up to the tropopause within a relatively short time. In a recent study, it has been demonstrated that concentrations of effective freezing nuclei can reach 0.1 $\mathrm{cm}^{-3}$ in the continental upper troposphere (Rogers et al., 1998). In view of such high numbers, it seems quite likely that heterogeneous freezing nuclei can control the formation and properties of SVCs, although homogeneous freezing nuclei are far more abundant.

Let me illustrate this important implication. In an air parcel ascending with $w=0.2 \mathrm{~cm} \mathrm{~s}^{-1}$ at $215 \mathrm{~K}$ only 0.0004 $\mathrm{cm}^{-3}$ ice particles form homogeneously (Fig. 3) sedimenting within a few minutes (Fig. 6), hence the SVC would only be short-lived. Assuming that $0.01 \mathrm{~cm}^{-3}$ effective freezing 
nuclei are present, all of them could be turned into smaller (by about a factor $(0.0004 / 0.01)^{1 / 3}=0.34$ ) ice particles at lower supersaturations that sediment less rapidly (by a factor of $0.34^{2}=0.12$ ), making the cloud event far more persistent. The assumed number of heterogeneous nuclei may be sufficient to prevent the liquid aerosols from ever freezing by competing more effectively for the available water vapor.

The spatial extent and frequency of occurrence of heterogeneously formed SVCs would be greater than in the case of the homogeneous formation mechanism, as low supersaturations occur more frequent and within larger atmospheric regions than higher values. It would be interesting to repeat this work including heterogeneous freezing to systematically study the relationship between number and freezing properties of the aerosols, the properties of the resulting SVCs, and $w$ and $T$. In a first step, the number and freezing threshold of potential nuclei could be open parameters, but ultimately more measurements are required to quantify them.

\section{Notation}

$\begin{array}{ll}a_{k}, b_{k} & \text { coefficients in Eq. (3) and (9) } \\ A & \text { total ice particle surface area density } \\ \alpha & \text { deposition coefficient of } \mathrm{H}_{2} \mathrm{O} \text { on ice } \\ D_{*} & \text { diffusion coefficient of } \mathrm{H}_{2} \mathrm{O} \text { molecules in air } \\ e & \text { saturation vapor number density over ice } \\ E & \text { volume extinction of cirrus clouds } \\ \lambda & \text { wavelength of light } \\ m & \text { total ice water mass } \\ n & \text { total number density of ice particles } \\ n_{a} & \text { total number density of aerosol particles } \\ Q & \text { Mie extinction efficiency } \\ r & \text { ice particle radius } \\ \hat{r} & \text { ice particle radius after the freezing event } \\ r_{S} & \text { smallest radius of a freezing aerosol particle } \\ r_{0} & \text { radius of a freezing aerosol particle } \\ s & \text { ice supersaturation } \\ S & \text { saturation ratio over a plane ice surface } \\ S_{\mathrm{cr}} & \text { freezing threshold saturation ratio } \\ t & \text { time after freezing event } \\ t_{g} & \text { growth timescale in the relaxation phase } \\ t_{S} & \text { sedimentation timescale } \\ t_{v} & \text { time after which developing SVCs become visible } \\ T & \text { air temperature } \\ v & \text { specific volume of a water molecule } \\ \bar{v} & \text { mean thermal speed of a water molecule } \\ w & \text { vertical velocity of an adiabatic air parcel } \\ & \end{array}$

Acknowledgements. This research was conducted within the project "Particles in the Upper Troposphere and Lower Stratosphere and Their Role in the Climate System" (PARTS) funded by the European Commission and contributes to the project "Particles and Cirrus Clouds" (PAZI) funded by the Hermann von HelmholtzGemeinschaft Deutscher Forschungszentren (HGF). I am grateful to two anonymous reviewers for making thorough and constructive comments on the original manuscript.

\section{References}

Beyerle, G., Schäfer, H.-J., Neuber, R., Schrems, O., and McDermid, I. S.: Dual wavelength lidar observation of tropical high altitude cirrus clouds during the ALBATROSS 1996 campaign, Geophys. Res. Lett., 25, 919-922, 1998.

Borrmann, S., Solomon, S., Dye, J. E., and Luo, B.: The potential of cirrus clouds for heterogeneous chlorine activation, Geophys. Res. Lett., 23, 2133-2136, 1996.

Chiou, E. W., McCormick, M. P., and Chu, W. P.: Global water vapor distributions in the stratosphere and upper troposphere derived from 5.5 years of SAGE II observations (1986-1991), J. Geophys. Res., 102, 19 105-19 118, 1997.

Dessler, A. E., Minschwaner, K., Weinstock, E. M., Hintsa, E. J., Anderson, J. G., and Russell, III, J. M.: The effects of tropical cirrus clouds on the abundance of lower stratospheric ozone, J. Atmos. Chem., 23, 209-220, 1996.

Gierens, K. M., Schumann, U., Helten, M., Smit, H., and Marenco, A.: A distribution law for relative humidity in the upper troposphere and lower stratosphere derived from three years of MOZAIC measurements, Ann. Geophysicae, 17, 1218-1226, 1999.

Goldfarb, L., Keckhut, P., Chanin, M.-L., and Hauchecorne, A.: Cirrus climatological results from lidar measurements at OHP (44ํN, $6^{\circ}$ E), Geophys. Res. Lett., 28, 1687-1690, 2001.

Hartmann, D. L., Holton, J. R., and Fu, Q.: The heat balance of the tropical tropopause, cirrus, and stratospheric dehydration, Geophys. Res. Lett., 28, 1969-1972, 2001.

Haynes, D. R., Tro, N. J., and George, S. M.: Condensation and evaporation of $\mathrm{H}_{2} \mathrm{O}$ on ice surfaces, J. Phys. Chem., 96, 8502-8509, 1992.

Hervig, M. and McHugh, M.: Cirrus detection using HALOE measurements, Geophys. Res. Lett., 26, 719-722, 1999.

Jensen, E. J., Toon, O. B., Pfister, L., and Selkirk, H. B.: Dehydration of the upper troposphere and lower stratosphere by subvisible cirrus clouds near the tropical tropopause, Geophys. Res. Lett., 23, 825-828, 1996.

Jensen, E. J., Toon, O. B., Vay, S. A., Ovarlez, J., May, R., Bui, P., Twohy, C. H., Gandrud, B., Pueschel, R. F., and Schumann, U.: Prevalence of ice-supersaturated regions in the upper troposphere: Implications for optically thin ice cloud formation, J. Geophys. Res., 106, 17 253-17 266, 2001.

Kärcher, B. and Solomon, S.: On the composition and optical extinction of particles in the tropopause region, J. Geophys. Res., 104, 27 441-27 459, 1999.

Kärcher, B. and Lohmann, U.: A parameterization of cirrus cloud formation: Homogeneous freezing of supercooled aerosols, J. Geophys. Res., 107, 10.1029/2001JD000470, 2002a.

Kärcher, B. and Lohmann, U.: A parameterization of cirrus cloud formation: Homogeneous freezing including effects of aerosol 
size, J. Geophys. Res., 107, in press, 2002b.

Kent, G. S., Winker, D. M., Osborn, M. T., and Skeens, K. M.: A model for the separation of cloud and aerosol in SAGE II occultation data, J. Geophys. Res., 98, 20 725-20 735, 1993.

Koop, Th., Luo, B. P., Tsias, A., and Peter, Th.: Water activity as the determinant for homogeneous ice nucleation in aqueous solutions, Nature, 406, 611-614, 2000.

Lelieveld, J., Bregman, A., Scheeren, H. A., Ström, J., Carslaw, K. S., Fischer, H., Siegmund, P. C., and Arnold, F.: Chlorine activation and ozone destruction in the northern lowermost stratosphere, J. Geophys. Res., 104, 8201-8213, 1999.

Lin, R.-F., Starr, D. O'C., DeMott, P. J., Cotton, R., Sassen, K., Jensen, E., Kärcher, B., and Liu, X.: Cirrus parcel model comparison project phase 1: The critical components to simulate cirrus initiation explicity, J. Atmos. Sci., 59, in press, 2002.

Lynch, D. K.: Subvisual cirrus: What it is and where you find it, in: Passive infrared remote sensing of clouds and the atmosphere, (Ed) Lynch, D. K., Society of Photooptical Instrumentation and Engineering (SPIE) Proceedings Series, Bellingham, WA, Vol. 1934, 264-274, 1993.

McFarquhar, G. M., Heymsfield, A. J., Spinhirne, J., and Hart, B.: Thin and subvisual tropopause tropical cirrus: Observations and radiative impacts, J. Atmos. Sci., 57, 1841-1853, 2000.

Meilinger, S. K., Kärcher, B., and Peter, Th.: On the impact of heterogeneous chemistry on ozone in the tropopause region, Geophys. Res. Lett., 28, 515-518, 2001.

Murphy, D. M., Kelly, K. K., Tuck, A. F., Proffitt, M. H., and Kinne, S.: Ice saturation at the tropopause observed from the ER-2 aircraft, Geophys. Res. Lett., 17, 353-356, 1990.

Ovarlez, J., van Velthoven, P., Sachse, G., Vay, S., Schlager, H., and Ovarlez, H.: Comparisons of water vapor measurements from POLINAT 2 with ECMWF analyses in high-humidity conditions, J. Geophys. Res., 105, 3737-3744, 2000.

Peter, Th., Luo, B. P., Kiemle, C., Flentje, H., Wirth, M., Borrmann, S., Thomas, A., Adriani, A., Cairo, F., Di Donfrancesco, G.,
Stefanutti, L., Santacesaria, V., Carslaw, K. S., and MacKenzie, A.R.: Ultrathin subvisible cirrus clouds at the tropical tropopause, in: 15th Int. Conf. Nucl. Atmos. Aerosols, American Institute of Physics, (Eds) Hale, B. N. and Kulmala, M., 619-622, 2000

Pruppacher, H. R. and Klett, J. D.: Microphysics of Clouds and Precipitation, Kluwer Acad. Publ., Dordrecht, 1997.

Rogers, D. C., DeMott, P. J., Kreidenweis, S. M., and Chen, Y.: Measurements of ice nucleating aerosols during SUCCESS, Geophys. Res. Lett., 25, 1383-1386, 1998.

Sassen, K. and Cho, B. S.: Subvisual-thin cirrus lidar dataset for satellite verification and climatological research, J. Appl. Meteorol., 31, 1275-1285, 1992.

Sassen, K. and Campbell, J. R.: A midlatitude cirrus cloud climatology from the facility for atmospheric remote sensing. Part I: Macrophysical and synoptic properties, J. Atmos. Sci., 58, 481496, 2001

Smith, J. B., Hintsa, E. J., Allen, N. T., Stimpfle, R. M., and Anderson, J. G.: Mechanisms for midlatitude ozone loss: Heterogeneous chemistry in the lowermost stratosphere?, J. Geophys. Res., 106, 1297-1309, 2001.

Solomon, S., Borrmann, S., Garcia, R. R., Portmann, R., Thomason, L., Poole, L. R., Winker, D., and McCormick, M. P.: Heterogeneous chlorine chemistry in the tropopause region, J. Geophys. Res., 102, 21 411-21 429, 1997.

Wang, P.-H., Minnis, P., McCormick, M. P., Kent, G. S., and Skeens, K. M.: A 6-year climatology of cloud occurrence frequency from Stratospheric Aerosol and Gas Experiment II observations (1985-1990), J. Geophys. Res., 101, 29407-29429, 1996.

Winker, D. M. and Trepte, C. R.: Laminar cirrus observed near the tropical tropopause by LITE, Geophys. Res. Lett., 25, 33513354, 1998.

Van De Hulst, H. C.: Light Scattering by Small Particles, John Wiley, New York, 1957. 